\title{
Cervical cancer screening (Pap
} testing) behaviours and acceptability of human papillomavirus self-testing among lesbian and bisexual women aged 21-26 years in the USA

\author{
Paul L Reiter, ${ }^{1}$ Annie-Laurie McRee ${ }^{2}$
}

\begin{abstract}
- Additional material is published online only. To view please visit the journal online (http://dx.doi.org/10.1136/jprhc2014-101004)

${ }^{1}$ Assistant Professor, Division of Cancer Prevention and Control, College of Medicine, The Ohio State University; Comprehensive Cancer Center, The Ohio State University; and College of Public Health, The Ohio State University, Columbus, OH, USA ${ }^{2}$ Assistant Professor, Comprehensive Cancer Center, The Ohio State University; and College of Public Health, The Ohio State University, Columbus, $\mathrm{OH}$, USA
\end{abstract}

\section{Correspondence to} Dr Paul L Reiter, Division of Cancer Prevention and Control, College of Medicine, The Ohio State University, Suite 525, 1590 North High Street, Columbus, OH 43201, USA; Paul.Reiter@osumc.edu

Received 19 June 2014 Revised 3 September 2014 Accepted 25 September 2014 Published Online First 10 November 2014

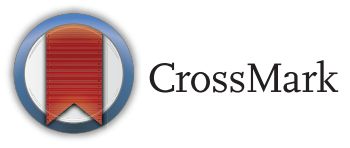

To cite: Reiter PL, McRee AL. J Fam Plann Reprod Health Care 2015;41:259-264.

\begin{abstract}
Objective Lesbian and bisexual women are at risk for human papillomavirus (HPV) infection and cervical disease. We examined cervical cancer screening (Pap testing) behaviours among these women and their acceptability of HPV selftesting at home, a potential cervical cancer screening strategy.

Methods We analysed data from a national sample of lesbian and bisexual women aged 21-26 years $(n=418)$ who completed our online survey in Autumn 2013. Logistic regression identified correlates of (1) having had a Pap test in the last 3 years and (2) willingness to use an HPV self-test at home.
\end{abstract}

Results About $70 \%$ of women had undergone a Pap test in the last 3 years. Pap testing was more common among women who had disclosed their sexual orientation to their health care provider [odds ratio $(O R)=2.01,95 \%$ confidence interval (CI) 1.02-3.95] and less common among women who self-identified as lesbian (OR=0.48, 95\% Cl 0.25-0.93). Just over half the women (51\%) were willing to use an HPV self-test at home. Women were more willing to use an HPV self-test at home if they were older $(\mathrm{OR}=1.16,95 \% \mathrm{Cl} 1.03-1.30)$ or reported higher levels of worry about getting an HPV-related disease $(O R=1.28,95 \% \mathrm{Cl}$ 1.01-1.63). The most common concerns about HPV self-testing at home were using the test incorrectly (70\%) and test accuracy (64\%).

Conclusions Many young lesbian and bisexual women have not had a recent Pap test. HPV self-testing at home may be a promising future strategy for reaching and screening these women. Findings highlight beliefs and concerns that could be addressed by self-test programmes.

\section{Key message points}

- About $30 \%$ of young lesbian and bisexual women in this study had not undergone recent cervical cancer screening (Pap testing).

- Over half the lesbian and bisexual women in this study were willing to use a human papillomavirus (HPV) selftest at home.

- Future HPV self-testing programmes should address the concerns about selftesting identified by women in this study.

\section{INTRODUCTION}

Persistent infection with oncogenic human papillomavirus (HPV) types causes virtually all cervical cancers. ${ }^{1}$ Cervical cancer is largely preventable through regular screening, ${ }^{2}$ yet more than 12000 new cases of cervical cancer and over 4000 deaths from cervical cancer are expected to occur among women in the USA during 2014. ${ }^{3}$ Current United States cervical cancer screening guidelines recommend that women aged 21-29 years receive cytology (i.e. Pap testing) every 3 years, and women aged 30-65 years receive a combination of cytology and HPV testing every 5 years (preferred strategy) or cytology every 3 years (acceptable strategy). ${ }^{4} \mathrm{HPV}$ testing is therefore currently recommended as a cervical cancer screening 'cotest', but there is interest in HPV testing as a primary screening option. 
Indeed, the US Food and Drug Administration (FDA) recently approved an HPV test as a primary cervical cancer screening option for women starting at age 25 years. $^{5}$

Given the current and potential future role of HPV testing in cervical cancer screening, it is important to examine HPV self-testing as a screening option for women. HPV self-tests involve women using a device on their own to collect a specimen for HPV testing. HPV self-tests are currently not licensed for use in the USA but have high enough accuracy to be considered as a viable screening strategy in the future. ${ }^{6}$ The sensitivity and specificity of HPV self-tests (sensitivity $=86 \%$, specificity $=81 \%$ ) for detecting cervical intraepithelial neoplasia Grade 2 or more severe compare favourably to liquid-based cytology (sensitivity $=81 \%$, specificity $=94 \%$ ) and physician-collected specimens for HPV testing (sensitivity $=97 \%$, specificity $=83 \%) .{ }^{6}$ In an attempt to reach women who have not had a recent Pap test, international studies have successfully mailed HPV self-tests to women at home and had them use and return a self-test device (up to $34 \%$ of women used and returned their HPV self-test). ${ }^{7}$ Less, however, is known about HPV selftesting at home among women in the USA, though most are willing to use an HPV self-test. ${ }^{8-10}$

Lesbian and bisexual women are at risk for HPV infection and cervical disease since HPV can be transmitted between female sexual partners ${ }^{11}$ and many of these women have current or past male sexual partners from whom they could have acquired HPV. ${ }^{12}$ Past research suggests that up to $30 \%$ of lesbian and bisexual women have a current genital HPV infection (about $43 \%$ of all women in the USA have a current infection). ${ }^{11} 13 \quad 14$ Furthermore, about $20 \%$ of young adult lesbian and bisexual women report a history of cervical abnormalities, which is comparable to young adult heterosexual women (also about 20\%). ${ }^{15}$ It is therefore recommended that all women, regardless of sexual orientation, follow the same cervical cancer screening recommendations. ${ }^{16}$

Although relatively few studies have examined cervical cancer screening among lesbian and bisexual women, ${ }^{15}$ 17-22 most have found that women who have sex with women, especially lesbians, are less likely to have had a recent Pap test compared to heterosexual women. ${ }^{15} 172021$ One potential explanation for this disparity in cervical cancer screening is that lesbian and bisexual women utilise sexual and reproductive health services (e.g. oral contraceptive services) less frequently than heterosexual women. ${ }^{17}$ Thus, HPV self-testing at home may be a promising strategy for reaching and screening lesbian and bisexual women for cervical cancer. However, no studies, to our knowledge, have examined acceptability of HPV self-testing at home among lesbian and bisexual women.

The current study examined Pap testing behaviours among a national sample of young lesbian and bisexual women. We also examined their willingness to use an HPV self-test at home and concerns they would have about using a self-test at home. The results will be highly relevant to future programmes aimed at increasing cervical cancer screening among lesbian and bisexual women.

\section{METHODS}

\section{Study design}

We conducted a cross-sectional study with individuals who were: (1) aged 18-26 years; (2) lived in the USA; and (3) self-identified as lesbian, gay, bisexual or transgender (LGBT). ${ }^{23}$ Participants were members of an online LGBT specialty panel that is a subset of the Harris Interactive Online Panel. Harris Interactive constructs this voluntary research panel through online and offline recruitment strategies throughout the USA. In exchange for completing multiple online surveys each month, panel members receive points that can later be redeemed for rewards. The Institutional Review Board at The Ohio State University approved the study.

Of 2014 panel members who were confirmed eligible for the study, 1005 (50\%) provided consent and completed our online survey in October and November 2013. In the present study we report data from women aged 21-26 years who self-identified as lesbian or bisexual $(n=418)$. We exclude data from 125 female respondents aged 18-20 years since cervical cancer screening is not currently recommended for females under the age of 21 years. Women included in the current study were from 48 states and the District of Columbia.

\section{Measures}

Outcome variables

We examined two main outcomes: (1) whether women had undergone a Pap test within the last 3 years (i.e. within the current recommended screening guidelines for this age range ${ }^{4}$ ); and (2) women's willingness to use an HPV self-test at home. Women were classified as having either undergone a Pap test in the last 3 years (i.e. a recent Pap test) or not. If women indicated they had not had a Pap test in this timeframe, a survey question then asked the main reason why they had not. Women could indicate only one main reason, and survey software rotated the order of response options. Among all women, we assessed how willing they would be to use an HPV self-test at home. We provided a brief description of the HPV self-test to women, indicating that it is a cervical cancer screening test that they would complete by themselves at home and mail the collected specimen to their doctor. Response options included "definitely not willing", "probably not willing", "not sure", "probably willing" and "definitely willing." We classified women as "willing" (definitely or probably willing: coded as 1) or "not willing" (all other 
responses: coded as 0). The survey then asked all women what concerns they would have about using an HPV self-test at home. Women could indicate multiple responses from a list of potential concerns.

Correlates

The survey assessed a wide range of demographic and health-related characteristics as potential correlates. We examined age at sexual debut, number of lifetime sexual partners, and history of any sexually transmitted infection (STI). We also assessed health insurance coverage, receipt of a routine check-up in past year, and if women had received any doses of HPV vaccine. Survey questions asked if women had disclosed their sexual orientation to their health care provider and whether they thought they had ever been discriminated against by a health care provider because of their sexual orientation, ${ }^{24}$ both of which are important issues for this population. ${ }^{25}$

The survey assessed knowledge and beliefs about HPV and HPV-related disease using items from our previous studies. ${ }^{26-28}$ We calculated an HPV knowledge score based on the proportion of correct responses to six true/false statements (possible range $=0-1)$. The survey assessed women's worry about getting HPV-related disease (1 item, possible range $=1-4)$, perceived severity of HPV-related disease ( 1 item, possible range $=1-4$ ) and perceived likelihood of cervical cancer ( 1 item, possible range $=1-4$ ). The survey also assessed whether women perceived a lower risk of cervical cancer compared to heterosexual women ( 1 item, possible range $=1-5)$. All continuous variables were coded so that higher values indicate greater levels of that construct.

\section{Data analysis}

We used logistic regression to identify correlates of our two main outcomes: (1) having had a Pap test in the last 3 years and (2) willingness to use an HPV self-test at home. For each outcome, we entered all statistically correlates $(p<0.05)$ from univariable models into a multivariable model to produce adjusted odds ratios (ORs) and 95\% confidence intervals (CIs). We used descriptive statistics to examine reasons why women had not had a Pap test in the last 3 years and women's concerns about using an HPV self-test at home. We used Chi square $\left(\chi^{2}\right)$ tests to determine if these outcomes differed between lesbian and bisexual women. We conducted all analyses in Stata IC V.13 ${ }^{\mathrm{TM}}$ (Statacorp, College Station, Texas, USA) using two-tailed tests and a critical $\alpha$ of 0.05 .

\section{RESULTS}

\section{Participant characteristics}

Most women were non-Hispanic white (68\%), not married or living with a partner $(60 \%)$ and reported a household income of less than $\$ 50000$ (69\%; see online Supplementary Table S1). About $73 \%$ of women self-identified as bisexual and $27 \%$ selfidentified as lesbian. Participants' mean age was 23.8 years [standard deviation $(\mathrm{SD})=1.7$ years]. About half the women $(52 \%)$ had a college degree. Women had moderate knowledge about HPV (mean $=0.62$, $\mathrm{SD}=0.28$ ) but tended to report low levels of worry about getting HPV-related disease (mean=1.8, $\mathrm{SD}=0.9)$ and perceived likelihood of cervical cancer (mean $=2.2, \mathrm{SD}=0.6$ ). Women generally believed that getting an HPV-related disease would be severe (mean $=3.5, \mathrm{SD}=0.7$ ).

\section{Pap testing}

Overall, 70\% (291/418) of women had undergone a Pap test within the last 3 years. Among those who had had a Pap test in the last 3 years, 67\% (195/291) had done so in the last year. About 24\% (101/418) of women had never had a Pap test. The most common main reasons why women had not had a Pap test in the last 3 years were cost (21\%), embarrassment (20\%), lack of a health care provider recommendation (13\%) and believing a Pap test is not necessary because they did not have any health problems (11\%). All other reasons were reported by less than $10 \%$ of women. Reasons for not having a Pap test in the last 3 years did not differ between lesbian and bisexual women (all $p>0.05$ ).

In multivariable analyses (see online Supplementary Table S2), Pap testing in the last 3 years was more common among women who were older (OR for 1 -year increase $=1.21,95 \%$ CI 1.02-1.43), had their own health insurance (OR=2.13, 95\% CI 1.09-4.16), had a routine check-up in the past year $(\mathrm{OR}=2.41$, 95\% CI 1.38-4.21), had received at least one dose of $\mathrm{HPV}$ vaccine $(\mathrm{OR}=2.63,95 \%$ CI $1.47-4.70)$ or had disclosed their sexual orientation to their health care provider $(\mathrm{OR}=2.01,95 \%$ CI 1.02-3.95). Compared to women who reported having no lifetime sexual partners, Pap testing in the last 3 years was more common among those who reported one to four sexual partners $(\mathrm{OR}=8.08,95 \% \mathrm{CI} 2.79-23.42)$ or five or more sexual partners $(\mathrm{OR}=17.06,95 \% \mathrm{CI}$ 5.62-51.77). Women who self-identified as lesbian $(\mathrm{OR}=0.48,95 \%$ CI $0.25-0.93)$ or were Hispanic (compared to non-Hispanic whites; $\mathrm{OR}=0.30,95 \%$ CI 0.14-0.63) were less likely to have had a Pap test in the last 3 years.

\section{HPV self-testing}

Just over half $(51 \% ; 212 / 418)$ the women were classified as willing to use an HPV self-test at home (24\% indicated "definitely willing" and $27 \%$ indicated "probably willing"). Of the $49 \%$ of women who were classified as not willing to use an HPV self-test at home, 11\% indicated "definitely not willing", 16\% indicated "probably not willing" and 22\% indicated "not sure". In multivariable analyses (see online Supplementary Table S3), women were more willing 
to use an HPV self-test at home if they were older (OR for 1-year increase $=1.16,95 \%$ CI $1.03-1.30$ ) or reported higher levels of worry about getting an HPV-related disease $(\mathrm{OR}=1.28,95 \%$ CI 1.01-1.63). Willingness to use an HPV self-test at home was nearly identical among women who had a Pap test within the last 3 years (51\%) and those who had not had a recent Pap test $(51 \%)(p=0.90)$.

The most common concerns women reported about using an HPV self-test at home were concerns about using the test incorrectly (70\%), the test might not be accurate (64\%), would rather go to a doctor to get screened for cervical cancer (44\%), the test might hurt $(25 \%)$, and would not want to return a completed test through the mail (23\%). All other concerns were reported by less than $10 \%$ of women. Concerns about using an HPV self-test at home did not differ between lesbian and bisexual women (all $p>0.05$ ).

\section{DISCUSSION}

Almost one-third of young adult lesbian and bisexual women in our study had not had a Pap test in the past 3 years, which is much higher than what a recent study showed for all women in the USA of comparable ages (16\%). ${ }^{29}$ Our finding therefore adds to the growing body of evidence of cervical cancer screening disparities among lesbian and bisexual women. ${ }^{15} 172021$ Many of the correlates of Pap testing identified among women in our study do, however, mirror those among women in the USA as a whole. We observed lower levels of recent Pap testing among Hispanic women and higher levels among women who were older, reported more lifetime sexual partners, had health insurance, and had received other preventive care in the past year. ${ }^{30} 31$

Recent Pap testing was less common among lesbian women compared to bisexual women, a finding similar to previous studies. ${ }^{15} 17$ There are several potential contributing factors to this finding, including lesbians lacking knowledge about screening, utilising health care service less frequently, and having no health insurance. ${ }^{17} 193233$ Relationships and communication between lesbian women and their health care providers also likely play an important role in determining whether they undergo Pap tests. Indeed, women in our study, regardless of sexual identity (lesbian or bisexual), who had disclosed their sexual orientation to their health care provider had greater odds of having had a recent Pap test. ${ }^{34}$ It may be that women who disclose their orientation are more comfortable with, have greater trust in, and communicate more effectively with their health care providers, which in turn makes it more likely that these women will undergo recommended care (e.g. Pap testing). Thus, it is key that health care providers do not make assumptions regarding women's sexual orientation, and that efforts are made to promote environments in which patients are comfortable communicating this information to their providers.

About one in five women reported that cost was the main reason they had not undergone a Pap test in the last 3 years and insuring oneself was a correlate of recent Pap testing in multivariable analyses. These findings are important since lesbian and bisexual women are less likely than heterosexual women to have health insurance coverage. ${ }^{35}$ Increased insurance coverage due to the Affordable Care Act may help women access routine preventive health care, ${ }^{36}$ and thus provide more opportunities for Pap testing among lesbian and bisexual women. However, to increase cervical cancer screening, it will also be necessary for future interventions to educate both lesbian and bisexual women and health care providers about the importance of screening for this population and address other reasons why some women are not receiving Pap tests. For example, lesbian and bisexual women may benefit from targeted education about the need for screening in the absence of health problems and strategies that could decrease potential embarrassment, such as HPV self-testing in the privacy of their own homes.

Just over half the women in our study were willing to use an HPV self-test at home. To our knowledge, this provides the first insight into lesbian and bisexual women's acceptability of HPV self-testing. Our finding is similar to those of previous studies showing many women in the USA are willing to use an HPV self-test at home. ${ }^{8-10}$ From a public health standpoint, HPV self-testing at home may be particularly important for lesbian and bisexual women since they utilise sexual and reproductive health services (including Pap testing) less frequently than heterosexual women. ${ }^{15} 172021$ Given the potentially increasing role of HPV testing in cervical cancer screening, ${ }^{5}$ future research should further explore the possibility of HPV self-testing for lesbian and bisexual women, especially those who have not had a recent cervical cancer screening test. One potential strategy for reaching this population may be an Internet-based HPV self-testing programme, which has been successful in promoting self-testing for other STIs. ${ }^{37}$ Future research should also examine how HPV self-testing may affect subsequent Pap testing and receipt of follow-up care (e.g. colposcopy), since it will be critical that women who complete HPV self-tests also engage in these other behaviours.

Consistent with previous studies, ${ }^{38-40}$ some of the most common concerns about using an HPV self-test at home were using the test incorrectly, test accuracy, perceived pain associated with performing the test, and not wanting to return a completed test through the mail. We believe that many of these concerns can be addressed by future interventions to promote HPV self-testing at home. For example, materials sent with HPV self-tests can include clear and 
easy-to-understand instructions for using the self-test device, discuss the accuracy of HPV self-testing, and alleviate concerns about self-tests being painful and returning the device through the mail. Materials should also provide information about HPV infection and HPV-related disease among lesbian and bisexual women. Providing this targeted information may increase women's level of worry about getting an HPV-related disease, which was positively associated with willingness to use an HPV self-test at home among women in our study. Thus, materials sent with HPV self-tests that address common concerns and target modifiable beliefs may offer a low-cost and sustainable strategy that should be explored by future HPV self-testing programmes.

Study strengths include a national sample of lesbian and bisexual women and the inclusion of a wide range of potential correlates. Limitations include selfreported Pap testing behaviours, which may be subject to recall bias. Our study included women who selfidentified as lesbian or bisexual, which may not include all women who have sex with women. Our analyses examined women aged 21-26 years, and HPV testing is currently recommended as a cervical cancer screening 'cotest' for women aged 3065 years. ${ }^{4}$ However, the FDA recently approved an HPV test as a primary cervical cancer screening option for women starting at age 25 years, ${ }^{5}$ suggesting that HPV testing may become a screening option for younger women in the future. Women's willingness to do a self-test at home may overstate self-testing in practice as intentions do not always translate into behaviour. Additional limitations include a modest response rate and a lack of data on non-respondents.

Many lesbian and bisexual women have not had a recent Pap test despite being at risk for HPV infection and HPV-related disease. HPV self-testing at home may be a promising strategy for increasing cervical cancer screening among this population. Concerns about selftesting and modifiable beliefs associated with willingness to use an HPV self-test at home identified in this study represent targets for future efforts promoting this potential cervical cancer screening strategy.

Funding Support provided by the National Cancer Institute at the National Institutes of Health (P30CA016058).

Competing interests Paul Reiter has received research grants from Merck Sharp \& Dohme Corporation and Cervical Cancer-Free America via an unrestricted educational grant from GlaxoSmithKline. These funds were not used to support this research study.

Ethics approval The Institutional Review Board at The Ohio State University approved the study.

Provenance and peer review Not commissioned; externally peer reviewed.

\section{REFERENCES}

1 Gillison ML, Chaturvedi AK, Lowy DR. HPV prophylactic vaccines and the potential prevention of noncervical cancers in both men and women. Cancer 2008;113(10 Suppl.): 3036-3046.

2 IARC Working Group on the Evaluation of Cancer-Preventive Strategies. IARC Handbooks of Cancer Prevention: Cervix Cancer Screening (Vol. 10). Lyon, France: IARC Press, 2005.

3 American Cancer Society. Cancer Facts \& Figures 2014. Atlanta, GA: American Cancer Society, 2014.

4 Saslow D, Solomon D, Lawson HW, et al. American Cancer Society, American Society for Colposcopy and Cervical Pathology, and American Society for Clinical Pathology screening guidelines for the prevention and early detection of cervical cancer. CA Cancer J Clin 2012;62:147-172.

5 US Food and Drug Administration. FDA approves first human papillomavirus test for primary cervical cancer screening. 2014. http://www.fda.gov/newsevents/newsroom/pressannouncements/ ucm394773.htm [accessed 2 September 2014].

6 Zhao FH, Lewkowitz AK, Chen F, et al. Pooled analysis of a self-sampling HPV DNA test as a cervical cancer primary screening method. J Natl Cancer Inst 2012;104: 178-188.

7 Racey CS, Withrow DR, Gesink D. Self-collected HPV testing improves participation in cervical cancer screening: a systematic review and meta-analysis. Can J Public Health 2013;104: e159-e166.

8 Richman AR, Brewer NT, Liebman AK, et al. Optimising human papillomavirus self-testing for high risk women. Sex Transm Infect 2011;87:118-122.

9 Scarinci IC, Litton AG, Garces-Palacio IC, et al. Acceptability and usability of self-collected sampling for HPV testing among African-American women living in the Mississippi Delta. Womens Health Issues 2013;23:e123-e130.

10 Vanderpool RC, Jones MG, Stradtman LR, et al. Self-collecting a cervico-vaginal specimen for cervical cancer screening: an exploratory study of acceptability among medically underserved women in rural Appalachia. Gynecol Oncol 2013;132(Suppl. 1):S21-S25.

11 Marrazzo JM, Koutsky LA, Stine KL, et al. Genital human papillomavirus infection in women who have sex with women. J Infect Dis 1998;178:1604-1609.

12 Marrazzo JM, Gorgos LM. Emerging sexual health issues among women who have sex with women. Curr Infect Dis Rep 2012;14:204-211.

13 Marrazzo JM. Genital human papillomavirus infection in women who have sex with women: a concern for patients and providers. AIDS Patient Care STDS 2000;14:447-451.

14 Hariri S, Unger ER, Sternberg M, et al. Prevalence of genital human papillomavirus among females in the United States, the National Health And Nutrition Examination Survey, 2003-2006. J Infect Dis 2011;204:566-573.

15 Charlton BM, Corliss HL, Missmer SA, et al. Reproductive health screening disparities and sexual orientation in a cohort study of U.S. adolescent and young adult females. J Adolesc Health 2011;49:505-510.

16 American Cancer Society. Cancer facts for lesbians and bisexual women. 2013. http://www.cancer.org/healthy/ findcancerearly/womenshealth/cancer-facts-for-lesbians-andbisexual-women [accessed 2 September 2014].

17 Agenor M, Krieger N, Austin SB, et al. Sexual orientation disparities in Papanicolaou test use among US women: the role of sexual and reproductive health services. Am J Public Health 2014;104:e68-e73.

18 Brown JP, Tracy JK. Lesbians and cancer: an overlooked health disparity. Cancer Causes Control 2008;19:1009-1020. 
19 Roberts SJ, Patsdaughter CA, Grindel CG, et al. Health related behaviors and cancer screening of lesbians: results of the Boston Lesbian Health Project II. Women Health 2004;39:41-55.

20 Kerker BD, Mostashari F, Thorpe L. Health care access and utilization among women who have sex with women: sexual behavior and identity. J Urban Health 2006;83:970-979.

21 Matthews AK, Brandenburg DL, Johnson TP, et al. Correlates of underutilization of gynecological cancer screening among lesbian and heterosexual women. Prev Med 2004;38:105-113.

22 Marrazzo JM, Koutsky LA, Kiviat NB, et al. Papanicolaou test screening and prevalence of genital human papillomavirus among women who have sex with women. Am J Public Health 2001;91:947-952.

23 Reiter PL, McRee AL, Katz ML, et al. HPV vaccination among young adult gay and bisexual men in the United States. Am J Public Health (in press).

24 Rainbow Health Initiative. Voices of health: a survey of LGBTQ health in Minnesota. 2013. http://www.rainbowhealth. org/resources-for-you/research-and-publications [accessed 2 September 2014].

25 Stevens PE, Hall JM. Stigma, health beliefs and experiences with health care in lesbian women. Image J Nurs Sch 1988;20:69-73.

26 Brewer NT, Gottlieb SL, Reiter PL, et al. Longitudinal predictors of human papillomavirus vaccine initiation among adolescent girls in a high-risk geographic area. Sex Transm Dis 2011;38:197-204.

27 Gilbert P, Brewer NT, Reiter PL, et al. HPV vaccine acceptability in heterosexual, gay, and bisexual men. Am J Mens Health 2011;5:297-305.

28 Reiter PL, McRee AL, Pepper JK, et al. Longitudinal predictors of human papillomavirus vaccination among a national sample of adolescent males. Am J Public Health 2013;103:1419-1427.

29 Centers for Disease Control and Prevention. Cancer screening - United States, 2010. MMWR Morb Mortal Wkly Rep 2012;61:41-45.

30 Centers for Disease Control and Prevention (CDC). Cervical cancer screening among women aged 18-30 years -
United States, 2000-2010. MMWR Morb Mortal Wkly Rep 2013;61:1038-1042.

31 Smith AM, Heywood W, Ryall R, et al. Association between sexual behavior and cervical cancer screening. J Womens Health (Larchmt) 2011;20:1091-1096.

32 Tracy JK, Lydecker AD, Ireland L. Barriers to cervical cancer screening among lesbians. $J$ Womens Health (Larchmt) 2010;19:229-237.

33 Gay and Lesbian Medical Association. Guidelines for Care of Lesbian, Gay, Bisexual, and Transgender Patients. 2008. http:// glma.org/_data/n_0001/resources/live/GLMA\%20guidelines $\%$ 202006\%20FINÄL.pdf [accessed 2 September 2014].

34 Tracy JK, Schluterman NH, Greenberg DR. Understanding cervical cancer screening among lesbians: a national survey. BMC Public Health 2013;13:442.

35 Buchmueller T, Carpenter CS. Disparities in health insurance coverage, access, and outcomes for individuals in same-sex versus different-sex relationships, 2000-2007. Am J Public Health 2010;100:489-495.

36 Collins SR, Garber T, Robertson R. Realizing Health Reform's Potential: How the Affordable Care Act is Helping Young Adults Stay Covered. New York, NY: The Commonwealth Fund, 2011.

37 Chai SJ, Aumakhan B, Barnes M, et al. Internet-based screening for sexually transmitted infections to reach nonclinic populations in the community: risk factors for infection in men. Sex Transm Dis 2010;37:756-763.

38 Anhang R, Nelson JA, Telerant R, et al. Acceptability of self-collection of specimens for HPV DNA testing in an urban population. J Womens Health (Larchmt) 2005;14:721-728.

39 Howard M, Lytwyn A, Lohfeld L, et al. Barriers to acceptance of self-sampling for human papillomavirus across ethnolinguistic groups of women. Can J Public Health 2009;100:365-369.

40 Giorgi Rossi P, Marsili LM, Camilloni L, et al. The effect of self-sampled HPV testing on participation to cervical cancer screening in Italy: a randomised controlled trial (ISRCTN96071600). Br J Cancer 2011;104:248-254. 